\title{
DIAGNÓSTICO NUTRICIONAL DE PACIENTES CIRÚRGICOS
}

\section{Nutritional diagnosis of surgical patients}

\author{
Celina de Azevedo DIAS, Maria Goretti Pessoa de Araújo BURGOS
}

ABCDDV/630

\begin{abstract}
Dias CA, Burgos MGPA. Diagnóstico nutricional de pacientes cirúrgicos. ABCD Arq Bras Cir Dig 2009;22(1):2-6
RESUMO - Racional - A desnutrição pode afetar pacientes cirúrgicos, aumentando o tempo de permanência hospitalar, a incidência de complicações pós-operatórias e retardo na cicatrização de feridas, levando ao aumento da morbimortalidade. Objetivo - Diagnosticar o estado nutricional de pacientes cirúrgicos. Método - Avaliaram-se 70 pacientes através de parâmetros antropométricos (peso, altura, Índice de Massa Corporal e percentual de perda de peso) e bioquímicos (albumina, hematócrito e hemoglobina) na admissão e em dois períodos do internamento hospitalar. Resultados - Na admissão detectarou-se maior percentual de desnutrição em idosos (32,4\%) e de excesso de peso em adultos $(33,4 \%)$. Na evolução ponderal durante os 15 dias de internamento, $88,6 \%$ teve perda de peso < 5\% e redução significativa do Índice de Massa Corporal, mas mantendo-se dentro da normalidade. Os pacientes com doença maligna apresentaram risco nutricional elevado na admissão e associação positiva com hipoalbuminemia. Constatou-se mais desnutridos nesta população quando se utilizou o risco nutricional através do percentual de perda de peso (63\%), em comparação com o Índice de Massa Corporal (38\%) e albuminemia isolada (17,1\%). Conclusões - 1- Não ocorreu perda de peso moderada ou grave no período estudado; 2 - na admissão e durante o internamento, a perda ponderal foi o melhor instrumento de avaliação do estado nutricional de pacientes cirúrgicos, quando comparada com o Índice de Massa Corporal e com a albumina; 3 - observou-se na admissão percentual significativo de sobrepeso em adultos e idosos.
\end{abstract}

DESCRITORES - Desnutrição. Estado nutricional. Cirurgia.

\section{INTRODUÇÃO}

A desnutrição é um dos problemas que afetam a América Latina e cerca de 50\% da população hospitalizada já é admitida com algum grau de desnutrição. Estudos realizados em países como os Estados Unidos, Suécia, Holanda, demonstraram que a desnutrição hospitalar não é exclusiva dos países em desenvolvimento, encontrando-se também nesses países prevalência entre 30 a $50 \%{ }^{12,14,20,24}$.

O Inquérito Brasileiro de Avaliação Nutricional (IBRANUTRI) avaliou quatro mil pacientes e demonstrou que aproxi $\neg$ madamente $48 \%$ da população hospitalizada eram desnutridos, destes $12,6 \%$ apresentavam desnutrição grave; observou-se também que a desnutrição foi maior com o aumento da permanência hospitalar e nos pacientes com neoplasia maligna ${ }^{23}$.

Sabe-se que a desnutrição pode afetar adversamente a evolução clínica dos pacientes por aumentar o tempo de permanência hospitalar, a incidência de complicações pós-operatórias, como infecções e retardo na cicatrização de feridas, além de aumentar a taxa de mortalidade ${ }^{2,3,12,14,22}$.

Neste contexto, a avaliação nutricional é de grande

Trabalho realizado no Hospital das Clínicas da Universidade Federal de Pernambuco - UFPE, Recife, PE, Brasil.

Endereço para correspondência: Maria Goretti Pessoa de Araújo Burgos, e-mail: gburgos@hotlink.com.br importância no período pré-operatório, tendo como objetivos principais definir o grau de desnutrição, identificar indivíduos em risco de desenvolver complicações decorrentes de déficits nutricionais e instituir precocemente a terapia nutricional especializada ${ }^{10}$.

Diante do exposto, as possíveis complicações relacionadas à desnutrição hospitalar em pacientes cirúrgicos motivaram este estudo, que teve como objetivo diagnosticar o estado nutricional de pacientes internados na clínica cirúrgica, de um hospital universitário do nordeste brasileiro.

\section{MÉTODO}

Este estudo do tipo série de casos foi realizado na Clínica de Cirurgia Digestiva do Hospital das Clínicas da Universidade Federal de Pernambuco (HC/UFPE), Recife, PE, Brasil. Foi aprovado pelo Comitê de Ética em Pesquisa envolvendo seres humanos da UFPE (CEP/CCS/UFPE), sob o ${ }^{\circ} 024 / 08$. Os pacientes foram informados sobre os objetivos da pesquisa, riscos e benefícios, bem como dos parâmetros a serem adotados e cada um deles assinou o termo de compromisso.

Dos 779 pacientes internados no período de abril a setembro de 2008, foram analisados 70 que atenderam aos critérios de inclusão da pesquisa. Participaram aqueles em período pré-operatório, de ambos os sexos, com idade $\geq 20$ anos, portadores de doenças do trato gastrintestinal, orientados ou acompanhados por pessoas que sabiam referir 
o peso habitual e permaneceram internados por período superior a sete dias. Excluíram-se adolescentes, gestantes, lactantes, pacientes que não saibam referir o peso habitual, com dificuldade de locomoção, com doenças em fase terminal; em pré e pós-operatório de transplante hepático e de operações bariátricas, além daqueles com infecção grave de feridas operatórias e sepse. Todos os dados foram obtidos das fichas de avaliação nutricional individual.

No diagnóstico nutricional, foram utilizados parâmetros antropométricos: peso atual, peso habitual, altura e Índice de Massa Corporal (IMC). O peso atual foi obtido utilizando balança plataforma da marca Filizola ${ }^{\mathrm{TM}}$ com capacidade de $150 \mathrm{Kg}$ e escala em divisões de $0,1 \mathrm{Kg}$. O peso habitual através da entrevista com o paciente, utilizando como referência a mudança do peso corporal após o surgimento da doença. A altura em metros detectada através do antropômetro de alumínio, acoplado à balança. O IMC foi calculado através da fórmula de Keys, et al. ${ }^{8}$, utilizando os parâmetros da Who ${ }^{25}$ para adultos e de Lipschitz ${ }^{11}$ para idosos. Todas as medidas antropométricas descritas foram realizadas pelo próprio pesquisador, a fim de minimizar erros. Como parâmetros bioquímicos foram avaliados albumina e hematócrito. Além disso, os pacientes foram classificados em dois níveis: com e sem risco nutricional através do percentual de perda ponderal do indivíduo após a doença, calculado entre o peso habitual e o atual dividido pelo peso habitual, durante os últimos seis meses ${ }^{1}$. A avaliação foi realizada até 72 horas de admissão hospitalar³. Observou-se a associação dos parâmetros antropométricos e bioquímicos com sexo, idade, tempo de internamento hospitalar e comorbidades.

Os dados foram agrupados no programa Excel e todas as análises realizadas pelo Statistical Package for the Social Sciences versão 13.021. Foram obtidas medidas estatísticas: média, desvio-padrão, variação (valor mínimo e valor máximo), distribuições absolutas e percentuais e os testes estatísticos: Mc-Nemar, t-Student para uma amostra; teste t-Student pareado, teste Qui-quadrado de Pearson ou Exato de Fisher quando as condições para utilização do teste Quiquadrado não foram verificadas. O nível de significância foi definido por $P<0,05$.

\section{RESULTADOS}

Foram admitidos na clínica cirúrgica, neste período, 779 pacientes, com permanência hospitalar média de seis dias, percentual de ocupação de $76,8 \%$ e ocorrência de 34 óbitos.

Do total de admitidos, $9 \%$ (70) foram incluídos na pesquisa a partir dos critérios estabelecidos, sendo $54 \%$ (38) do sexo masculino e $46 \%$ (32) do feminino, com idade de $55,66 \pm 14,77$ anos (24-83). Dentre os homens, 50\% deles eram idosos, enquanto nas mulheres $43 \%$ eram adultas.

Todos os pacientes apresentavam doenças gastrintestinais na fase inicial do diagnóstico que, para comparações estatísticas, foram divididas em grupo 1 (doença benigna) e grupo 2 (doença maligna) (Tabela 1).
TABELA 1 - Distribuição da amostra segundo o diagnóstico dos pacientes estudados na Clínica de Cirurgia Digestiva do HC/UFPE, 2008

\begin{tabular}{ll}
\hline VARIÁVEL & CASUÍSTICA (n, \%) \\
\hline DIAGNÓSTICO & \\
Grupo 1 (Doença benigna) & \\
Vias biliares & $14(45,2)$ \\
Fístulas gastrintestinais & $4(12,9)$ \\
Megaesôfago chagásico & $4(12,9)$ \\
Miscelânea & $9(29,0)$ \\
\hline TOTAL & $\mathbf{3 1}(\mathbf{1 0 0 , 0 )}$ \\
\hline Grupo 2 (Doença maligna) & \\
Neoplasia de reto & $12(30,8)$ \\
Neoplasia gástrica & $11(28,2)$ \\
Neoplasia de vias biliares & $5(12,8)$ \\
Outros & $11(28,2)$ \\
\hline TOTAL & $\mathbf{3 9}(\mathbf{1 0 0 , 0 )}$ \\
\hline
\end{tabular}

Quanto ao IMC, não foram observadas alterações significativas entre sete e 15 dias de internamento $(P=0,425)$, não diferindo também entre grupos etários (adultos e idosos), demonstrando na admissão percentual de $61,1 \%$ de eutróficos e apenas 5,6\% de desnutridos nos adultos; por outro lado, nos idosos, detectou-se $32,4 \%$ de desnutrição e $26,5 \%$ de excesso de peso.

Em relação aos parâmetros bioquímicos, denotou-se normalidade na albumina na maioria dos adultos $(4,02$ $\pm 0,54 \mathrm{mg} / \mathrm{dL})$ e idosos $(3,78 \pm 0,60 \mathrm{mg} / \mathrm{dL})$, diferentemente da hemoglobina e do hematócrito que sinalizaram maior anemia em ambos os grupos (Hb: adultos $=58,3 \%$ e idosos $=52,9 \%$; Ht: adultos $=61,1 \%$ e idosos $=52,9 \%$ ). Nos idosos, ocorreu redução significativa do hematócrito $(P=0,021)$ com sete dias de internamento $(35,00 \pm 5,20 \%$ para 33,34 $\pm 4,95 \%)$.

Na evolução ponderal durante a hospitalização, grande parte dos pacientes teve perda de peso $<5 \%(88,6 \%)$, compatível com desnutrição leve (Tabela 2).

TABELA 2 - Percentual de perda de peso do início da doença à admissão e durante o internamento hospitalar dos pacientes da Clínica de Cirurgia Digestiva do HC/ UFPE, 2008

\begin{tabular}{llll}
\hline Variável & Admissão & $\mathbf{7}^{\mathbf{0}} \mathbf{D I H}$ & $\mathbf{1 4}^{\circ} \mathbf{D I H}$ \\
\hline Perda de peso (\%) - n (\%) & & & \\
$<5$ & $25(35,7)$ & $65(92,9)$ & $31(88,6)$ \\
5 a 10 & $14(20,0)$ & $4(5,7)$ & $4(11,4)$ \\
$>10$ & $31(44,3)$ & $1(1,4)$ & - \\
Perda peso (\%)-Média \pm DP & $2,09 \pm 0,90$ & $1,09 \pm 0,33$ & $1,11 \pm 0,32$ \\
Valor de P & $\mathrm{P}(1)<0,001^{*}$ & $\mathrm{P}(2)<0,001^{*}$ & $\mathrm{P}(3)<0,001^{*}$ \\
\hline
\end{tabular}

$\mathrm{DIH}=$ Dia de Internamento Hospitalar; $*$ = diferença significante a 5,0\%. P (1)= resultado do teste entre o peso habitual e a avaliação na admissão; $\mathrm{P}(2)=$ resultado do teste entre a admissão e o $7^{\circ} \mathrm{DIH} ; \mathrm{P}(3)=$ resultado do teste entre o $7^{\circ}$ e o $14^{\circ} \mathrm{DIH}$

Comparando os momentos de avaliação desde a admissão, observa-se a manutenção do IMC (eutróficos) tanto nos 
adultos como nos idosos, apesar de diferenças estatísticas $(\mathrm{P} \leq 0,05)$ dentro das classificações de normalidade.

Não foram observadas mudanças no perfil bioquímico nos dois grupos etários, com exceção da albumina, que reduziu significativamente $(P=0,03)$ com sete dias nos adultos, todavia mantendo-se dentro dos valores normais $(4,02 \pm 0,54 \mathrm{mg} / \mathrm{dL}-3,89 \pm 0,59 \mathrm{mg} / \mathrm{dL})$. Além disso, apenas o Grupo 2 teve associação positiva com a hipoalbuminemia $(P=0,034)$.

Nos três períodos analisados, não houve diferenças entre o risco nutricional entre as distintas doenças; ao mesmo tempo, ficou evidente que o Grupo 2 apresentou risco nutricional elevado na admissão (Tabela 3), onde todos os pacientes com neoplasia gástrica e de vias biliares apresentaram perda ponderal superior a $10 \%$ desde o início da doença $(P=0,006)$. Constatou-se percentual maior de desnutridos nesta população, quando se utilizou o risco nutricional através do percentual de perda de peso $(63 \%)$, em comparação com o IMC (38\%) e com a albumina isolada $(17,1 \%)$.

TABELA 3 - Distribuição do risco nutricional através do percentual de perda de peso na admissão segundo o tipo do diagnóstico dos pacientes da Clínica de Cirurgia Digestiva do HC/UFPE, 2008

\begin{tabular}{lllll}
\hline \multirow{2}{*}{ Diagnóstico } & \multicolumn{2}{l}{ Risco Nutricional } \\
& $\begin{array}{l}\text { Com risco } \\
\text { n (\%) }\end{array}$ & $\begin{array}{l}\text { Sem risco } \\
\text { n (\%) }\end{array}$ & $\begin{array}{l}\text { Total } \\
\text { N (\%) }\end{array}$ & Valor de P \\
\hline Grupo 1 (Doença benigna) & & & & \\
Vias biliares & $6(42,9)$ & $8(57,1)$ & 14 & $\mathrm{P}(1)=0,948$ \\
Fístulas gastrintestinais & $2(50,0)$ & $2(50,0)$ & 4 & \\
Megaesôfago chagásico & $2(50,0)$ & $2(50,0)$ & 4 & \\
Miscelânea & $5(55,6)$ & $4(44,4)$ & 9 & \\
\hline Total & $\mathbf{1 5 ( 4 8 , 4 )}$ & $\mathbf{1 6 ( 5 1 , 6 )}$ & $\mathbf{3 1 ( 1 0 0 , 0 )}$ & \\
\hline Grupo 2 (Doença maligna) & & & & \\
Neoplasia de reto & $5(41,7)$ & $7(58,3)$ & 12 & $\mathrm{P}(1)=0,004^{*}$ \\
Neoplasia gástrica & $11(100,0)$ & - & 11 & \\
Neoplasia de vias biliares & $5(100,0)$ & - & 5 & \\
Outros & $8(72,7)$ & $3(27,3)$ & 11 & \\
\hline Grupo total & $\mathbf{2 9 ( 7 4 , 4 )}$ & $\mathbf{1 0}(\mathbf{2 5 , 6 )}$ & $\mathbf{3 9 ( 1 0 0 , 0 )}$ & \\
\hline
\end{tabular}

*= Diferença significante a 5,0\%. P (1)= através do teste Exato de Fisher

\section{DISCUSSÃO}

São escassos os trabalhos que analisam a prevalência de desnutrição em pacientes cirúrgicos, apesar de grandes pesquisas evidenciarem alta frequência de desnutrição no âmbito hospitalar².

A classificação dos pacientes na admissão quanto ao risco nutricional e sua associação com o tipo e severidade da doença, detectou $63 \%$ do grupo total com risco nutricional, sendo $74 \%$ no Grupo 2, fato comprovado por Conde et al. 2 em $57 \%$ e por Waitzberg, et al. ${ }^{23}$ em $66,9 \%$ neste grupo de doentes.

Sabe-se que a perda involuntária de peso prediz a existência de complicações no pós-operatório, onde o risco de complicações aumenta com a quantidade de peso perdido e com a velocidade desta perda ${ }^{2}$. Segundo DeWys, et al. ${ }^{4}$, a perda ponderal moderada a grave pode ocorrer de $40-80 \%$ em pacientes oncológicos, dependendo do tipo do tumor, o que ficou comprovado no presente trabalho nos tumores do trato gastrintestinal com variações de 42 a $100 \%$ de redução de peso.

Durante os três períodos avaliados no internamento, a perda ponderal parece ser o melhor parâmetro para identificar a desnutrição neste tipo de doentes, resultados em conformidade com Pereira, et al. ${ }^{17}$ e Dock-Nascimento, et al. ${ }^{5}$, ao avaliar neoplasia do aparelho digestivo.

Outro fator importante para determinar o estado nutricional no pré-operatório é a correlação de dados antropométricos com o tipo de diagnóstico, o que neste grupo não ficou demonstrado na associação tanto das doenças benignas como nas malignas $(P>0,05)$, provavelmente pelo reduzido tamanho da amostra.

Avaliando o risco nutricional segundo o tipo de diagnóstico, encontrou-se nas doenças malignas risco mais acentuado e marcante naqueles com neoplasia gástrica, afetando em menor grau os de neoplasia de reto, corroborando com os resultados de DeWys, et al. ${ }^{4}$ e Conde, et $\mathrm{al}^{2}$.

Quanto aos valores de albumina sérica, detectou-se redução significativa apenas na avaliação do sétimo dia de internamento e exclusivamente em adultos, diferentemente dos resultados de Mello, et al. ${ }^{13}$ que encontraram valores de albumina em torno de $3,4 \mathrm{mg} / \mathrm{dL}$ em pacientes cirúrgicos no Hospital das Clínicas de Porto Alegre, e Dock-Nascimento, et al. ${ }^{5}$ com valores de $3,1 \mathrm{mg} / \mathrm{dL}$ sem diferença estatística entre os grupos de doença maligna e benigna. Dados de Conde, et al. ${ }^{2}$ revelam que $49 \%$ de pacientes cirúrgicos com neoplasia digestiva apresentaram hipoalbuminemia, superior aos achados neste presente estudo; mas, ainda bastante superiores aos resultados de Kyle, et al. ${ }^{10}$ com $13 \%$ em indivíduos clínicos e cirúrgicos da Europa; na Bahia, resultados de instituição filantrópica revelam 35,3\% de hipoalbuminemia nas duas clínicas ${ }^{19}$. A diferença significativa encontrada entre a concentração sérica de albumina nos indivíduos com doença maligna e benigna $(P=0,034)$ discordou com os resultados de Dock-Nascimento, et al. ${ }^{5}$, que não encontraram diferença nos níveis de albumina; por outro lado, os valores de hematócrito e hemoglobina não mostraram diferenças entre os grupos.

Existem controvérsias quanto ao uso da albumina como marcador do estado nutricional, pela sua baixa sensibilidade principalmente no diagnóstico da fase aguda da desnutrição, por sua meia vida biológica ser relativamente longa ( \pm 20 dias), podendo transcorrer várias semanas para que ocorra resposta às variações na ingestão dietética e protéica, além de fatores não nutricionais poderem afetar seus valores, como o que pode ser visto na infecção, nos processos inflamatórios, trauma cirúrgico, lesão tecidual, enteropatias exsudativas, jejum prolongado para exames e/ ou operações, além do stress fisiológico de exames ${ }^{16}$, o que talvez justificasse sua redução em curto período.

Em trabalho recente ${ }^{18}$, realizado em idosos clínicos hospitalizados, observou-se através da hemoglobina que a prevalência de anemia era de $36,7 \%$, valores inferiores aos encontrados nesta pesquisa $(52,9 \%)$. Ao mesmo tempo, ocorreu redução estatisticamente significativa do 
hematócrito entre o início e fim do internamento ( $P=0,018)$, o que não foi comprovado ao estratificar a amostra em adultos e idosos. A anemia em doentes maiores de 60 anos e aumento da mortalidade são demonstrados por diversos autores ${ }^{6,7,9}$. Sabe-se que concentrações elevadas de hemoglobina estão associadas com a diminuição no tempo de internação e de custos hospitalares ${ }^{15}$. Além disso, existem algumas evidências que a anemia está associada com perda de peso em pacientes internados ${ }^{7}$. Em relação ao hematócrito, não foram encontrados dados em pacientes cirúrgicos para comparação com os achados desta pesquisa.

Em relação às medidas antropométricas, ficou demonstrado que houve redução significativa do IMC nos adultos ao comparar a primeira e terceira avaliação, ao contrário dos idosos, onde esta redução foi importante apenas no primeiro e segundo período. Porém, o IMC permaneceu dentro da normalidade em todo o internamento, provavelmente pela adequada terapia nutricional adotada, com até três dias de internação; além da baixa sensibilidade do IMC para detecção da DEP. Os valores de IMC desta pesquisa foram semelhantes aos encontrados por Kyle, et al. ${ }^{10} \mathrm{e}$ Manzanares, et al. ${ }^{12}$. Do mesmo modo, os primeiros 10 revelam redução do IMC e incremento da perda ponderal com o tempo de hospitalização.

No paciente cirúrgico eletivo, a assistência integral deve começar na admissão hospitalar através da avaliação nutricional, identificando pacientes desnutridos e com risco nutricional, otimizando assim os resultados da conduta terapêutica e dietética durante a internação e alta hospitalar.

Portanto, mais estudos de avaliação nutricional com associação de vários índices para o diagnóstico nutricional, com tamanho maior de amostra e diagnósticos variados são necessários, a fim de estabelecer condutas dietoterápicas adequadas, minimizando possíveis complicações da desnutrição.

\section{CONCLUSÃO}

A desnutrição foi mais prevalente em idosos e o excesso de peso em adultos, porém sem perda de peso grave, no internamento. $\mathrm{Na}$ admissão e hospitalização, a perda ponderal foi o melhor parâmetro para avaliar o estado nutricional de pacientes cirúrgicos, quando comparada com o IMC e com a albumina isolada, devendo ser utilizada como rotina na avaliação nutricional nestes pacientes.

Dias CA, Burgos MGPA. Nutritional diagnosis of surgical patients. ABCD Arq Bras Cir Dig 2009;22(1):2-6

ABSTRACT - Background - Malnutrition can affect surgical patients by increasing length of hospital stay, the incidence of postoperative complications and delayed wound healing, in addition to higher mortality rate. Aim - To diagnose nutritional status of surgical patients. Methods - Seventy patients were evaluated by anthropometric parameters (weight, height, Body Mass Index and percentage of weight loss) and biochemical (albumin, hemoglobin and hematocrit) in admission and in two periods of hospitalization. Results - On admission malnutrition percentage was higher in the elderly (32.4\%) and excess weight in adults (33.4\%). Weight during the 15 days of hospitalization presented $88.6 \%$ weight loss and significant reduction of Body Mass Index, while remaining within the normal range. Patients with malignant disease had higher nutritional risk in admission, positive association with hypoalbuminemia and weight loss (63\%), Body Mass Index (38\%) and albumin (17.1\%). Conclusions - 1. There was no severe weight loss throughout the hospitalization; 2 - on admission and during the hospital stay, the weight loss was the best tool for assessing the nutritional status of surgical patients, compared with Body Mass Index and the albumin.

HEADINGS - Malnutrition. Nutritional status. Surgery.

\section{REFERÊNCIAS}

1. Blackburn GL, Bristian BR. Nutritional and metabolic assessment of the hospitalized patient. JPEN. 1977;1:11-22.

2. Conde LC, López TF, Blanco PN, Delgado JA, Correa JJV, Lorenzo FFG. Prevalencia de desnutrición en pacientes con neoplasia digestiva previa cirugía. Nutr Hosp. 2008;23(1):46-53.

3. Correia MI, Waizberg DL. The impact of malnutrition on morbidity, mortality, length of hospital stay and costs evaluated through a multivariatemodel analysis. Clin Nutr. 2003;22:219-20.

4. Dewys WD, Begg C, Lavin PT, Band PR, Bennett JM, Bertino JR, Cohen MH, Douglass HO Jr, Engstrom PF, Ezdinli EZ, Horton J, Johnson GJ, Moertel CG, Oken MM, Perlia C, Rosenbaum C, Silverstein MN, Skeel RT, Sponzo RW, Tormey DC. Prognostic effect of weight loss prior to chemotherapy in cancer patients. Eastern Cooperative Oncology Group. Am J Med. 1980 Oct;69(4):4917.

5. Dock-Nascimento DB, Aguilar-Nascimento JE, Balster MMS. Índice de massa corporal e peso teórico subestimam o diagnóstico de desnutrição em pacientes cirúrgicos. Rev Bras Nutr Clin. 2005;20(4):251-4.

6. Ezekowitz JA, McAlister FA, Armstrong PW. Anemia is common in heart failure and is associated with poor outcomes. Insights from a cohort of 12,065 patients with new-onset heart failure. Circulation 2003;107:223-5.
7. Horwich TB, Fonarow GC, Hamilton MA, MacLellan WR, Borenstein J. Anemia is associated with worse symptoms, greater impairment in functional capacity and a significant increase in mortality in patients with advanced heart failure. J Am Coll Cardiol. 2002;39:1780-6.

8. Keys A, Fidanza F, Karvonen MJ, Kimura, N, Taylor HL. Indices of relative weight and obesity. J Chronic Dis. 1972;25:329-43.

9. Kosiborod M, Smith GL, Radford MJ, Foody JM, Krumholz HM. The prognostic importance of anemia in patients with heart failure. Am J Med. 2003;114:112-9.

10. Kyle UG, Pirlich M, Schuetz T, Lochs H, Pichard C. Is nutritional depletion by nutritional risk index associated with increased length of hospital stay? A population-based study. JPEN. 2004; 28:99-104.

11. Lipschitz DA. Screening for nutritional status in the elderly. Prim car. 1994;21: 55-67.

12. Manzanares W, Azcúnaga MF, Barreiro T, González M, Gelós C, Alejandro S, Nacaroto S, Techera A, Viquer S, Pittaluga A, Pittini G, Fariña G, Cancela M. Desnutrición asociada a enfermedad en los pacientes quirúrgicos del Hospital de Clínicas. Montevideo - Uruguay. Rev Bras Nutr Clin. 2005; 20(4):209-14.

13. Mello ED, Beghetto MG, Teixeira LB, Luft VC. Desnutrição hospitalar cinco anos após o IBRANUTRI. Rev Bras Nutr Clin 2003; 18(2):65-9. 
14. Menéndez AM. Farmacoterapia: Interacciones en $\neg$ tre los medicamentos y los nutrientes en Ia nutrición enteral. In: Guastavino P, Guida R, editores. Manual Fundamentos y estrategias del apoyo nutricional. Editado por Abbott Laboratories Argentina SA, Bue $\neg$ nos Aires; 2000. p. 203-10.

15. Nordyke RJ, Kim JJ, Goldberg GA, Vendiola R, Batra D, McCamish M, Thomasson JW. Impact of anemia on hospitalization time, charges, and mortality in patients with heart failure. Value Health. 2004 Jul-Aug;7(4):464-71.

16. Oñate-Ocaña LF, Aiello-Crocifoglio V, Gallardo-Rincón D, Herrera-Goepfert R, Brom-Valladares R, Carrillo JF, Cervera E, Mohar-Betancourt A. Serum albumin as a significant prognostic factor for patients with gastric carcinoma. Ann Surg Oncol. 2007 Feb;14(2):381-9.

17. Pereira SF, Aguilar-Nascimento JE. Composição corporal na desnutrição causada por câncer e doenças benignas do aparelho digestivo. Rev Col Bras Cir. 1998; 26(1):7-10.

18. Ramel A, Jonsson PV, Bjornsson S, Thorsdottir I. Anemia, nutritional status, and inflammation in hospitalized elderly. Nutr. 2008:1-7.

19. Rezende IFB, Oliveira VS, Kuwano EA, Leite APB, Rios I, Dórea YS, Chaves VL. Prevalência da desnutrição hospitalar em pacientes internados em um hospital filantrópico em Salvador (BA), Brasil. R Ci Méd biol. 2004;3:194-200.
20. Scattolin MAA, Avela GN, Toledo JCF, Yamaroto FW, Alves ER, Dias Neto VS. Avaliação nutricional de Idosos Internados no CHS: perfil nutricional à internação e correlação com escala de depressão e mini-mental. Rev Fac de Ciên Med Sorocaba 2005;7(1):15-20.

21. Statistical Package for the Social Science for Windows. SPSS/Pc version 13.0, Chicago: SPSS; 2003

22. Waitzberg DL. Repercussão da desnutrição hospitalar na evolução clínica de pacientes hospitalizados. Rev Bras Med. 2006; 63: 38-9.

23. Waitzberg DL, Caiaffa WT, Correia ITD. Hospital malnutrition: the Brazilian national survey (IBRANUTRI): a study of 4000 patients. Nutr. 2001;17(78):573-80.

24. Warterlow JC. Protein-energy malnutrition: the nature and extent of the problem. Clin Nutr. 1997;16 (Suppl): 3-9.

25. WHO. Physical status: the use and interpretation of anthropometry: report of a WHO expert committee. WHO Technical Report Series. Genebra, 1995.

Fonte de financiamento: não há Conflito de interesse: não há Recebido para publicação: 04/07/2008 Aceito para publicação: 13/10/2008 